\title{
PUBLISHER'S NOTE \\ THE PHENOMENOLOGY OF RIGHT HANDED NEUTRINOS
}

\author{
[INT. J. MOD. PHYS. E, Vol. 22, No. 8 (2013) 1330019] \\ MARCO DREWES \\ Physik Department T\%0, Technische Universität München, \\ James Franck Straße 1, D-85748 Garching, Germany \\ marco.drewes@tum.de
}

Published 12 September 2013

This paper was published online on 23 August 2013 without color coding in Appendix A table (page 55) and with misprints of Greek symbols in Appendix B (pages 56 and 57). These errors have been corrected in both the online and print versions of Int. J. Mod. Phys. E Vol. 22, No. 8 (2013) issue. 